\title{
The Effect of Manipulation of Neural Activity of Dorsolateral Prefrontal Cortex on Cognitive Flexibility Deficit
}

\author{
Amin Rezanejad Asl ${ }^{*}$, Ali Issazadegan, Farzaneh Mikaeli Manei
}

Department of Psychology, Faculty of Educational Sciences, University of Urmia, Urmia, Iran

\section{A BSTRACT}

Introduction: Deficit in cognitive flexibility has been observed in several mental and brain disorders. The dorsolateral prefrontal cortex is the most important brain region involved in cognitive flexibility related tasks. Using a computer model of neuronal stimulation, the aim of this study was to investigate the effects of manipulating neural activity in this brain region on cognitive flexibility. Materials and Methods: A computer simulation approach was used. Simulation was in the context of Leabra cognitive architecture and by using PBWM model was implemented. Results: This study shows that increasing neural activity percent in the dorsal lateral prefrontal cortex linearly decreased cognitive flexibility. In addition, reduction of neural activity in this region of the brain decreased cognitive flexibility in a larger extent. Conclusion: These data indicate that hypo- or hyperactivity of inhibitory interneurons in the dorsal lateral prefrontal cortex can cause deficit in cognitive flexibility. Manipulation of neural activity in this region can distinguish two subgroups of patients with impaired cognitive flexibility.

\section{Key words:}

1. Interneurons

2. Pliability

3. Prefrontal Cortex

*Corresponding Author: Amin Rezanejad Asl

E-mail:a.rezanejadasl@gmail.com 
تأثير دستكارى فعاليت عصبى قشر ييش ييشانى جانبى -خلفى بر كمبود انعطاف يذيرى شناختى

$$
\text { امين رضانزاد اصل"، على عيسى زادَان، فرزانه ميكائيلى منيع }
$$

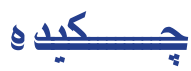

مقدمه: كمبود انعطافيذيرى شناختى در جندين اختين دئلال روانى و مغز مشاهده شده است. قشر

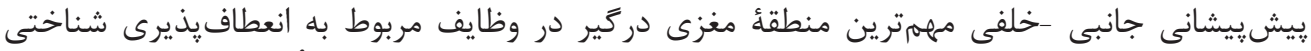

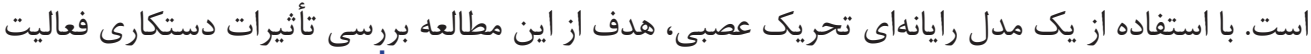

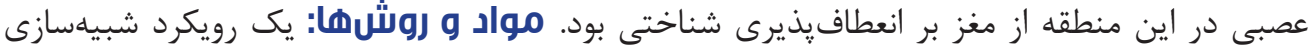

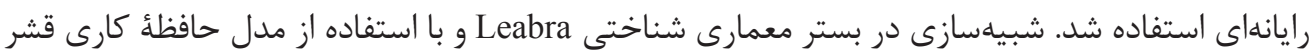

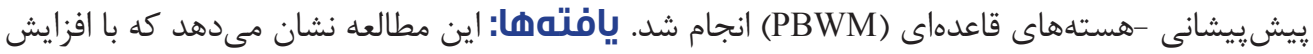

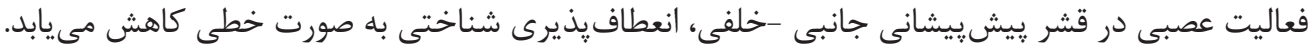

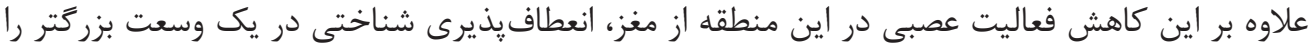

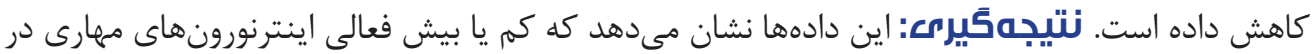

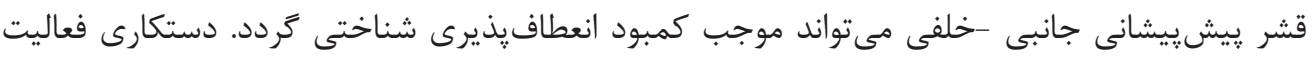

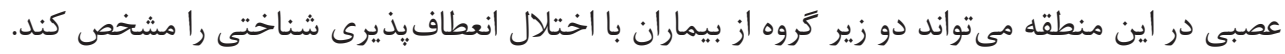

كليد وازهها:

ا إينترنورون

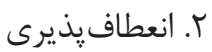

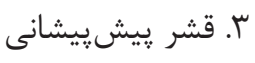

" نويسنده مسئول: امين رضانزاد اصل a.rezanejadasl@gmail.com آدرس الكترونيكى 


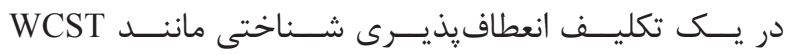

بيـان كننــد (9).

همجٍنيــن ســازو كار عصبــى دخيــل در ايــن فراينـــد نيــز

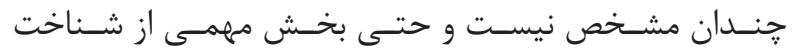

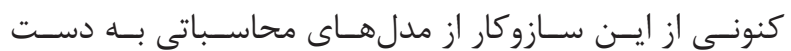

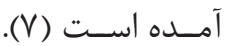

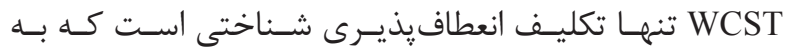

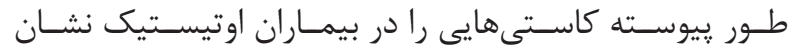

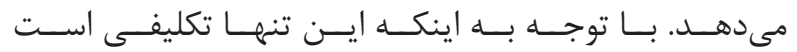

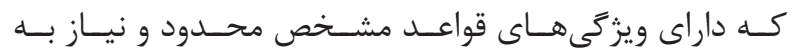

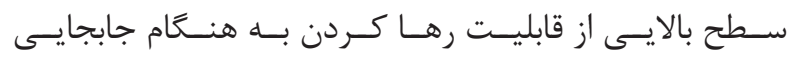

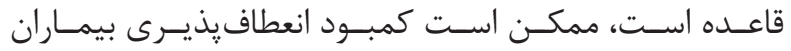

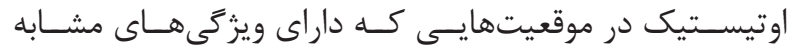

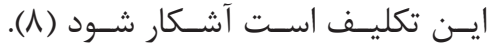

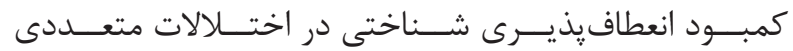

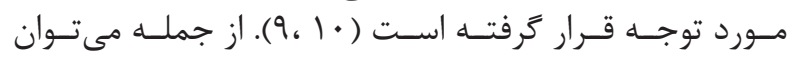

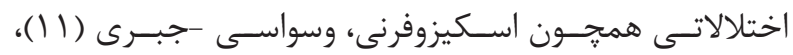

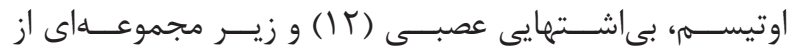

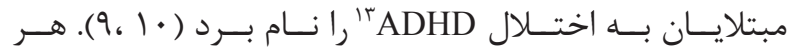

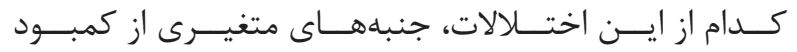

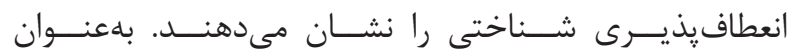

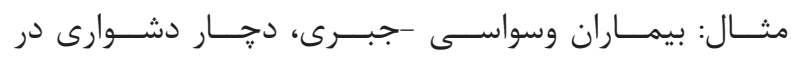

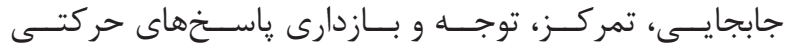

هســتند (T) (I).

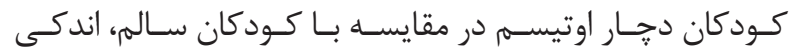

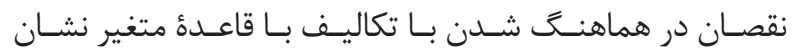

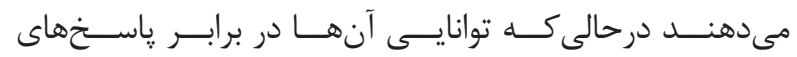

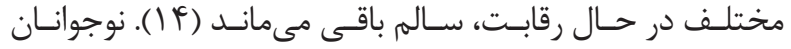

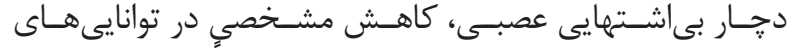

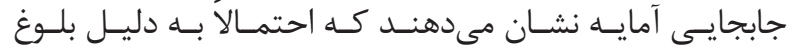

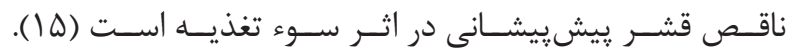

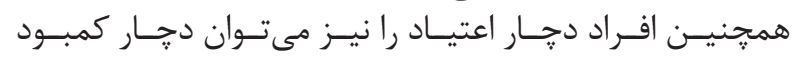

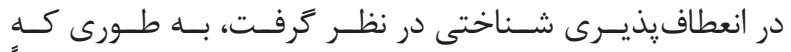

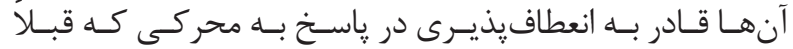

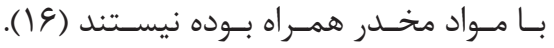

در تحقيقـى ميــزان فعاليــت مغــزى بيمـــاران دجـــار

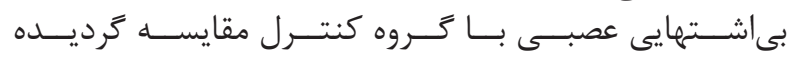

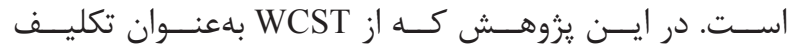

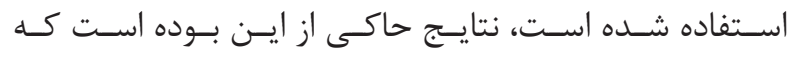

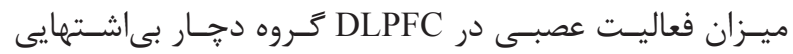

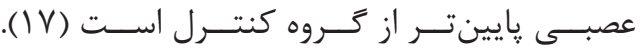

${ }^{1}$ Cognitive flexibility

${ }^{2}$ Shifting

${ }^{3}$ Mental set shifting

${ }^{4}$ Switching

${ }^{5}$ Wisconsin card sorting test

${ }^{6}$ Perseverative

${ }^{7}$ Functional magnetic resonance imaging

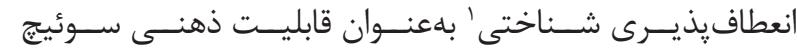

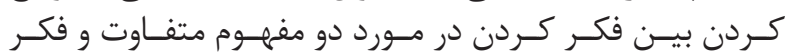

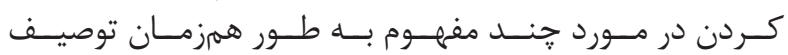

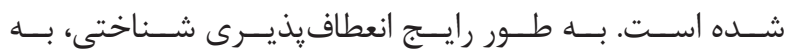

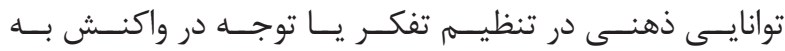

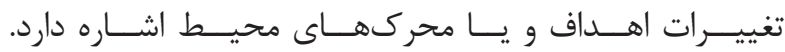

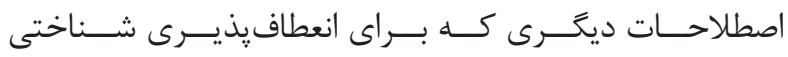

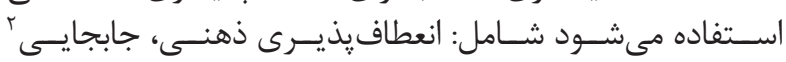

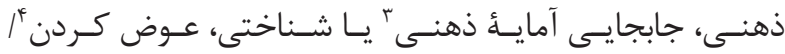

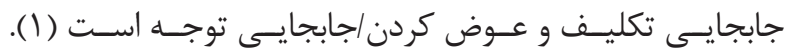

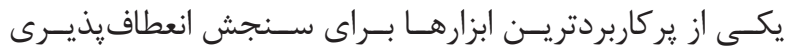

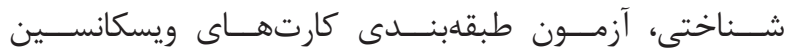

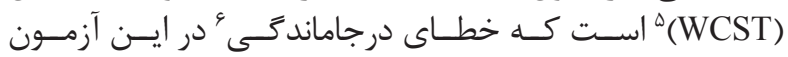

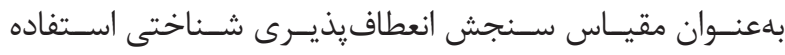

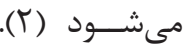

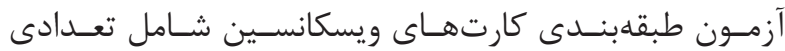

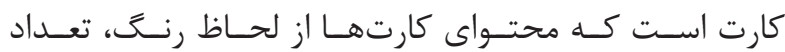

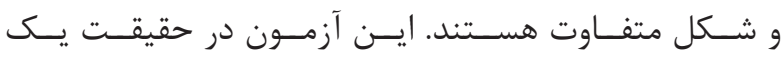

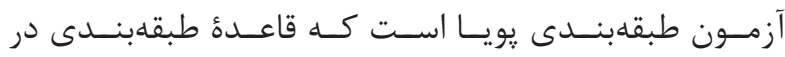

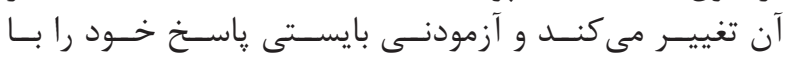

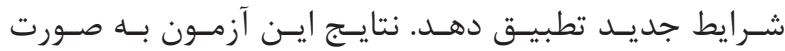

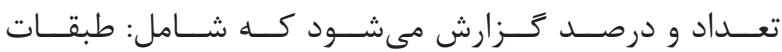

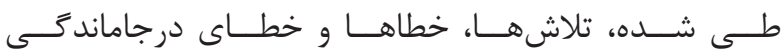

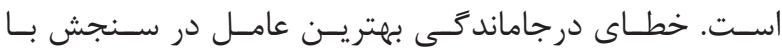

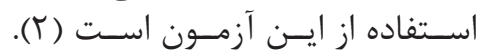

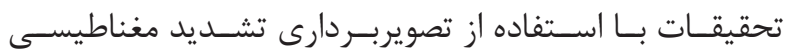

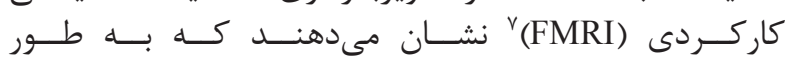

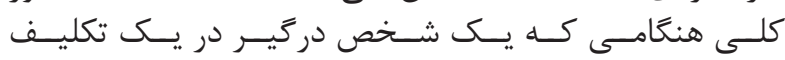

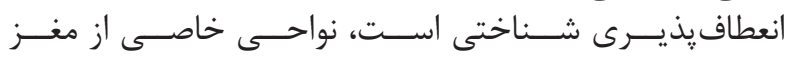

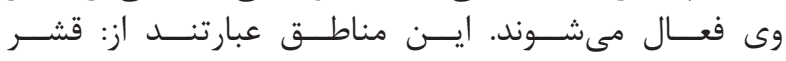

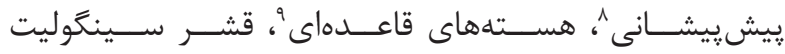

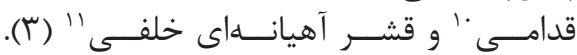

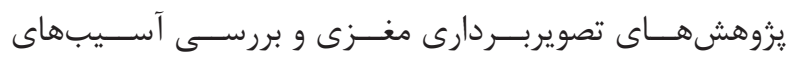

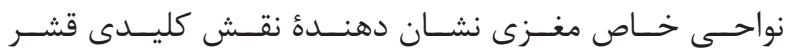

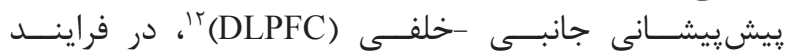

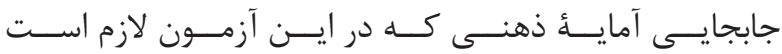

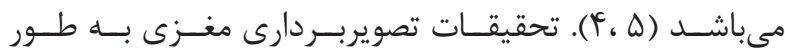

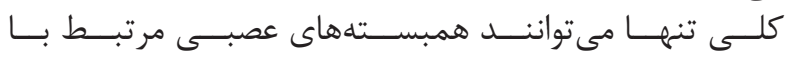

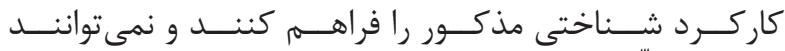

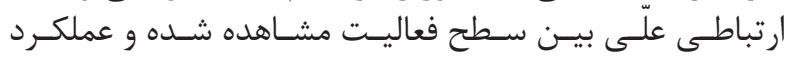

${ }^{8}$ Prefrontal cortex

${ }^{9}$ Basal ganglia

${ }^{10}$ Anterior cingulate cortex

${ }^{11}$ Posterior parietal cortex

${ }^{12}$ Dorsolateral prefrontal cortex

${ }^{13}$ Attention deficit hyperactivity disorder 


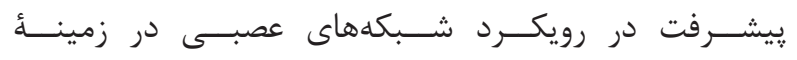

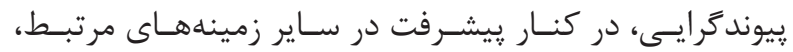

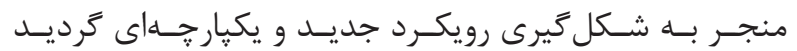

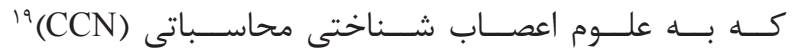

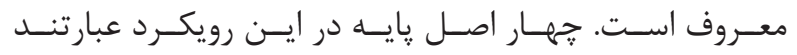

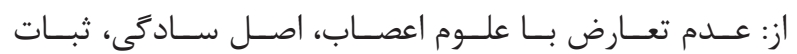

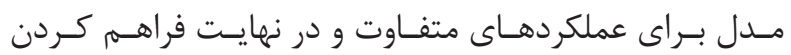

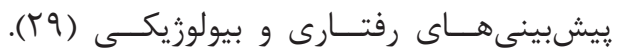

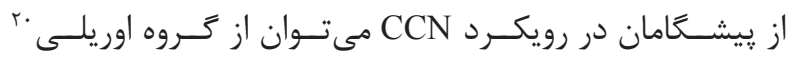

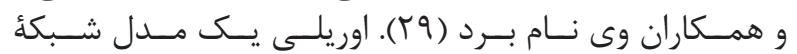

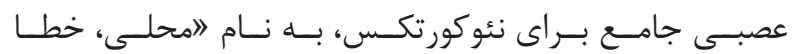

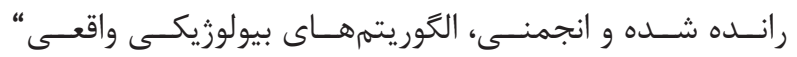

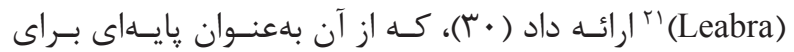

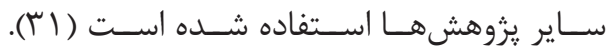

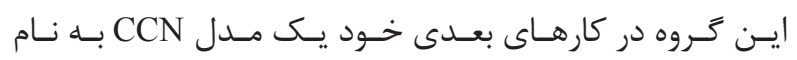

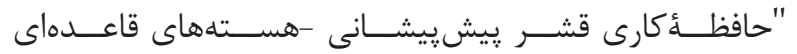
(PBWM)

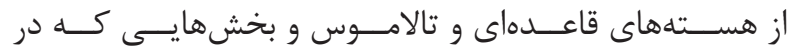

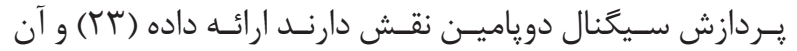

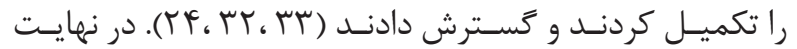

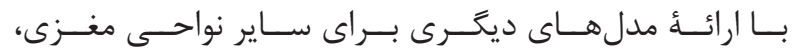

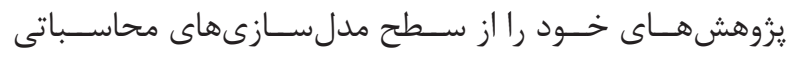

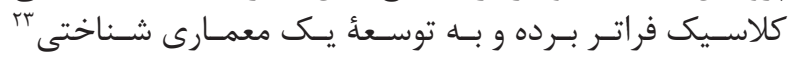

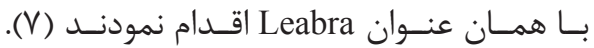

سيســتم PBWM در معمــارى شــناختى Leabra ورود،

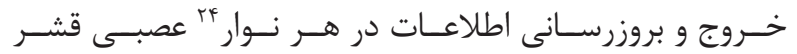

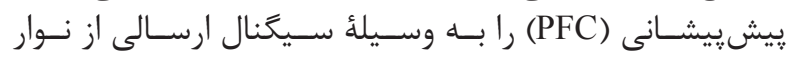

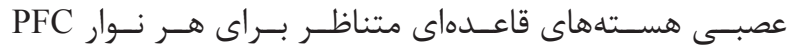

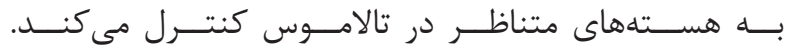

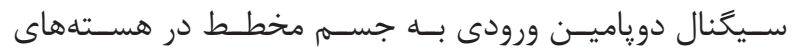

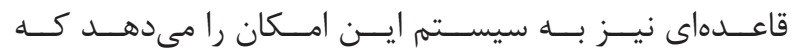

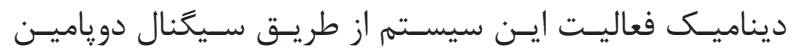

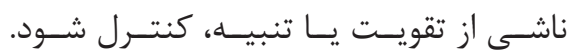

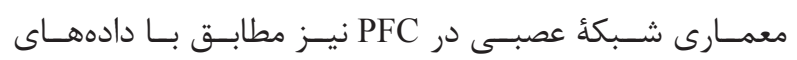

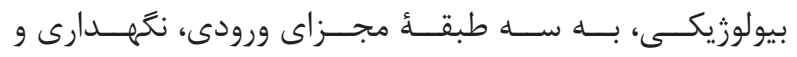

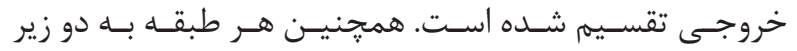

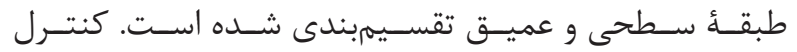

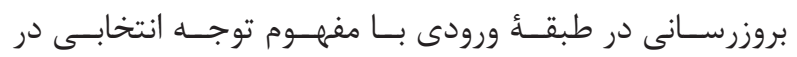

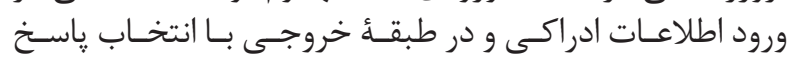

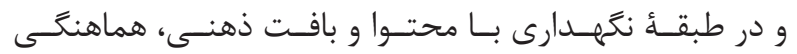
مفهومسى دارد (سب).

\footnotetext{
${ }^{14}$ Caudate nucleus

${ }^{15}$ Computer modeling

${ }^{16}$ Mathematical psychology

${ }^{17}$ Connectionism

${ }^{18}$ Emergent

${ }^{19}$ Computational cognitive neuroscience
}

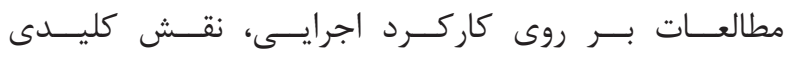

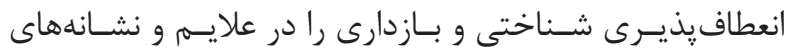

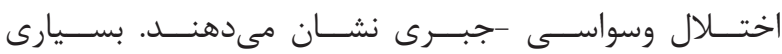

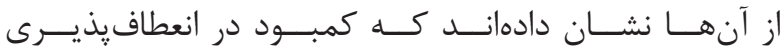

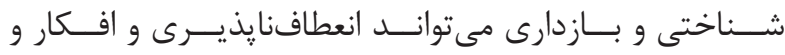

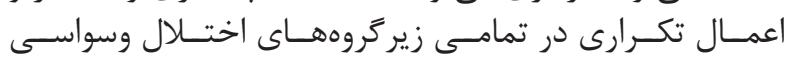

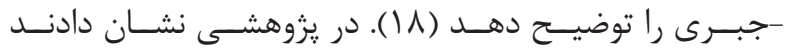

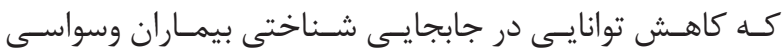

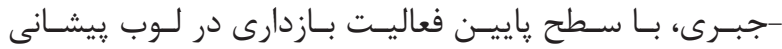

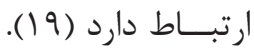

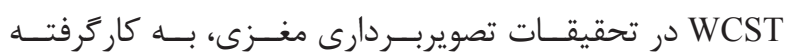

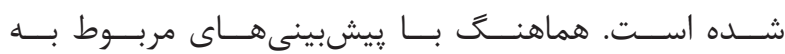

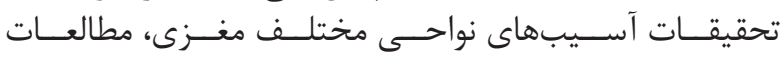

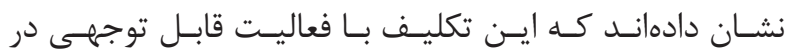

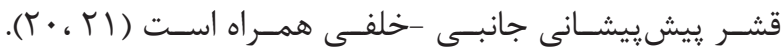

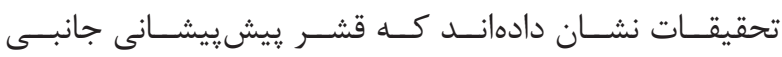

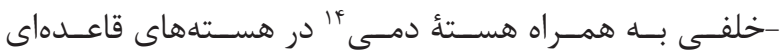

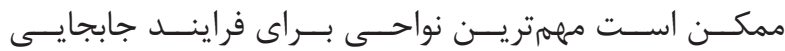

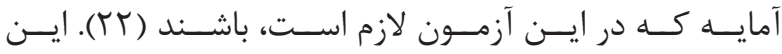

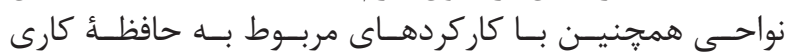

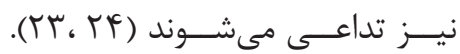

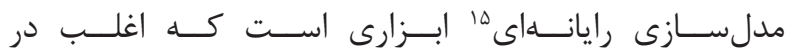

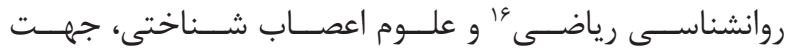

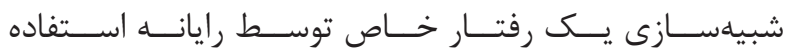

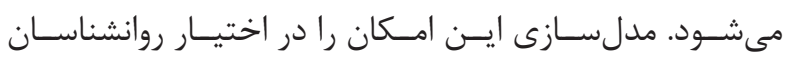

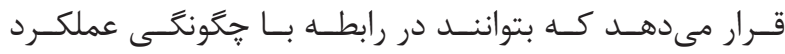

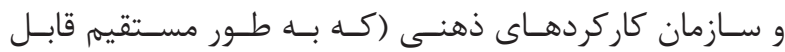

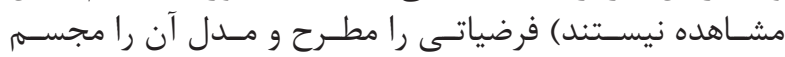

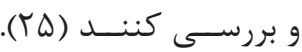

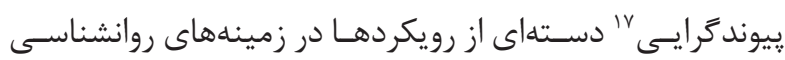

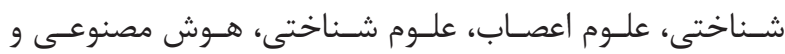

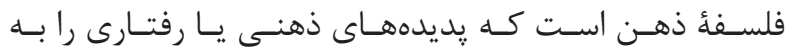

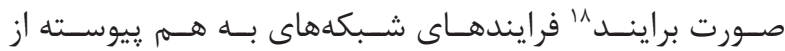

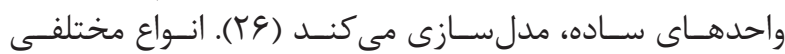

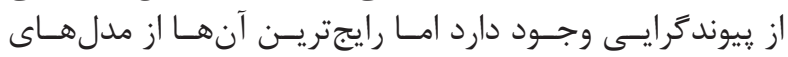

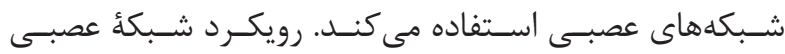

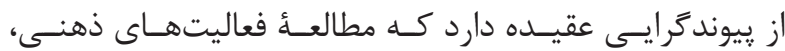

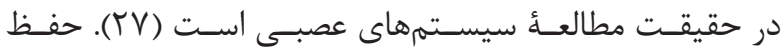

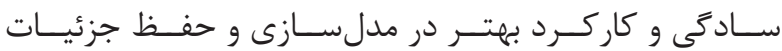

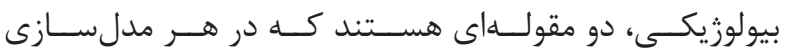

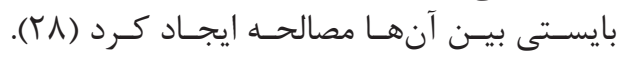

${ }^{20}$ O'Reilly

${ }^{21}$ Local, error-driven and associative, biologically realistic algorithm

${ }^{22}$ Prefrontal cortex basal ganglia working memory

${ }^{23}$ Cognitive architecture

${ }^{24}$ Stripe 


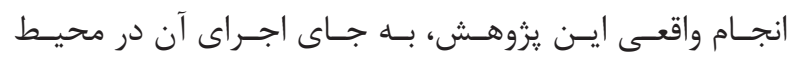

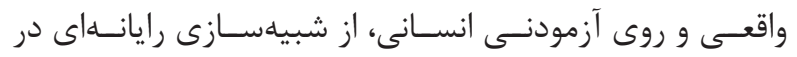

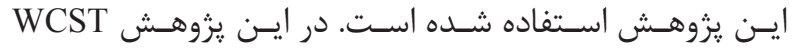

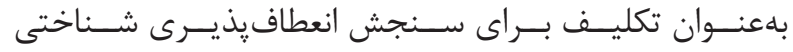

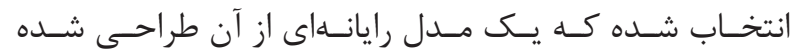

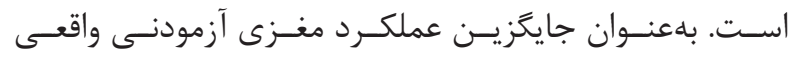

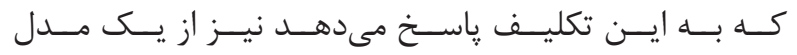

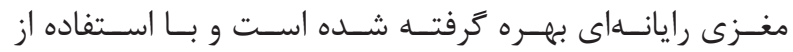

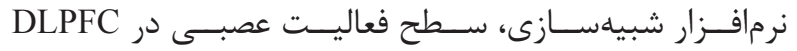

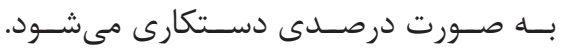

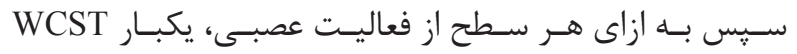

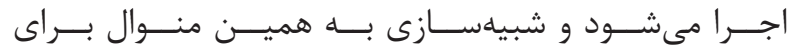

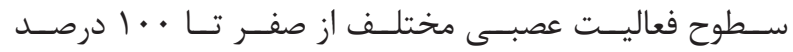

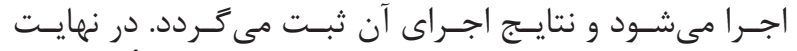

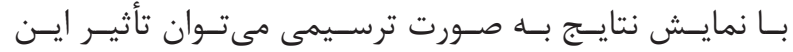

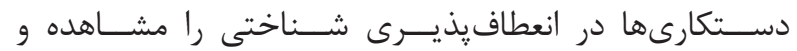

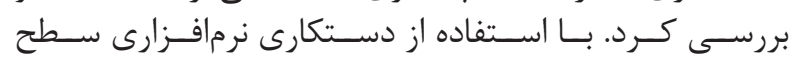

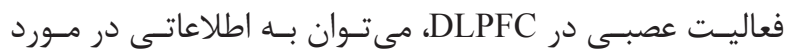

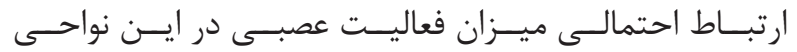

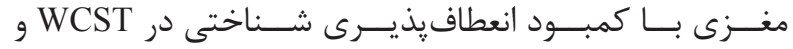

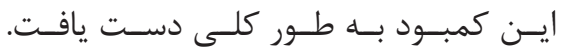

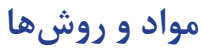

Emergent

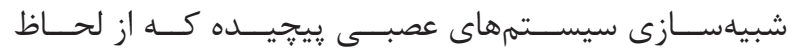

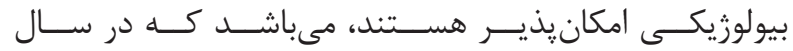

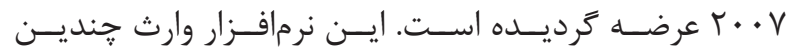

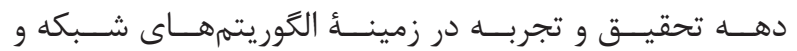

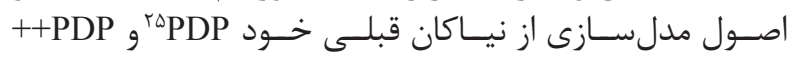

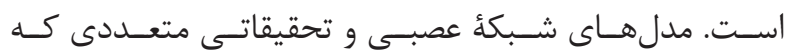

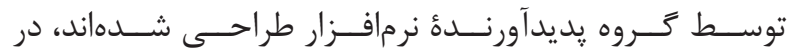

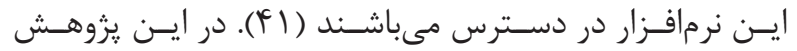

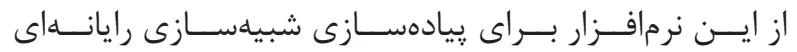

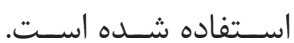

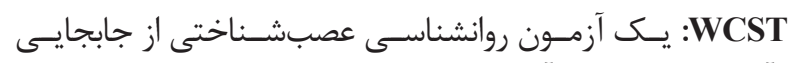

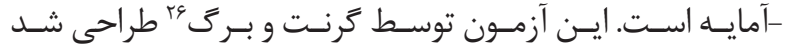

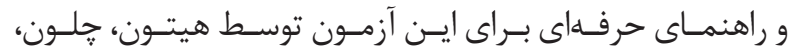

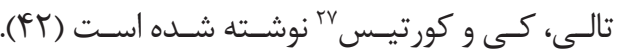

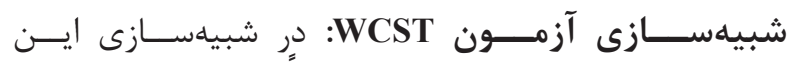

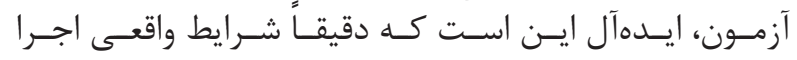

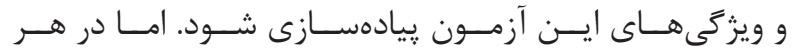

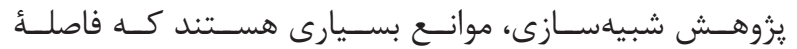

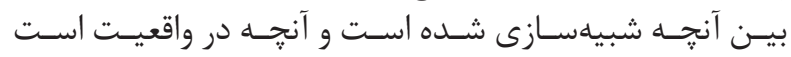

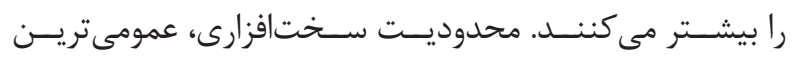

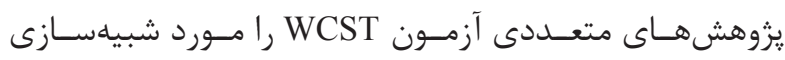

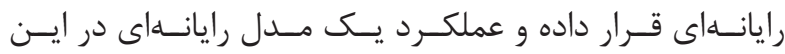

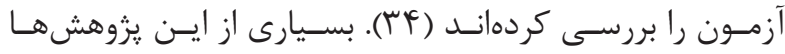

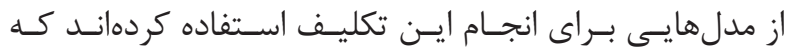

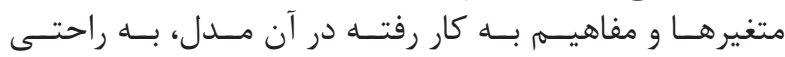

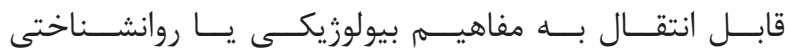

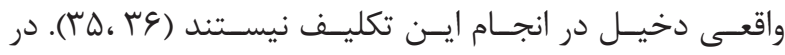

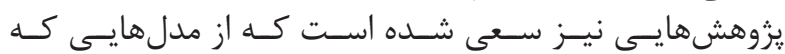

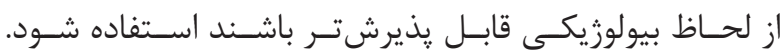

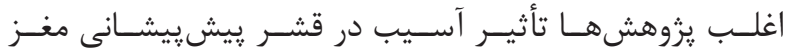

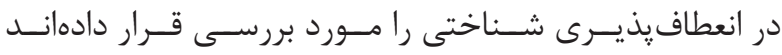

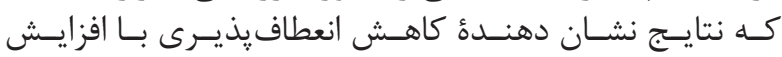

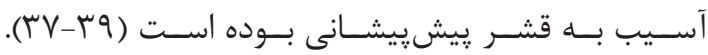

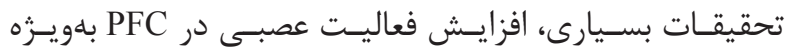

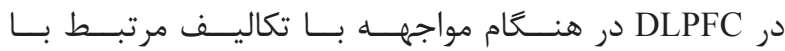

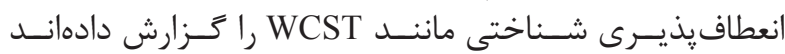

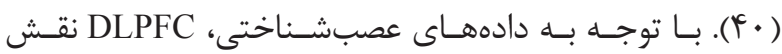

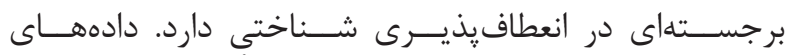

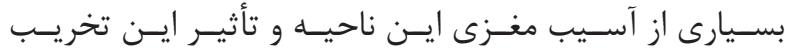

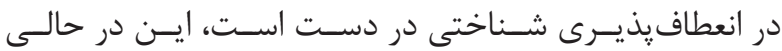

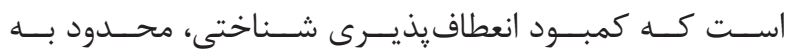

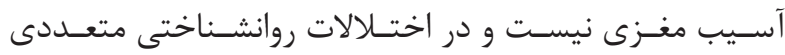

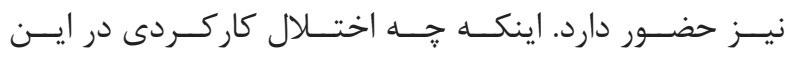

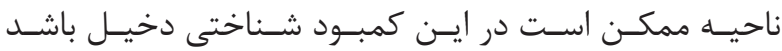

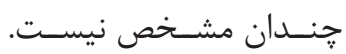

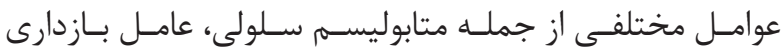

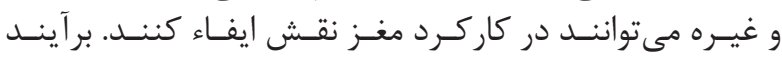

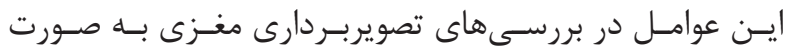

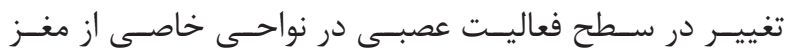

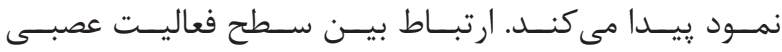

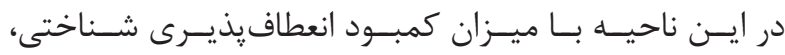

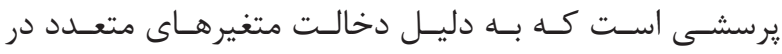

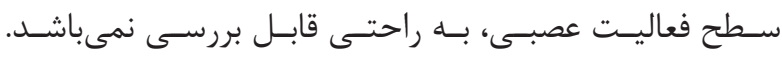

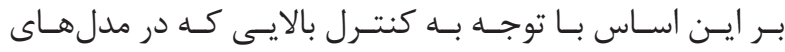

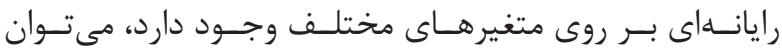

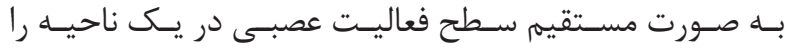

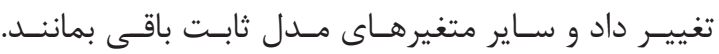

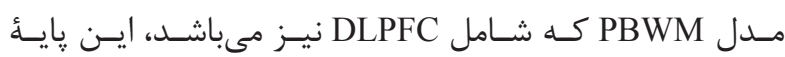

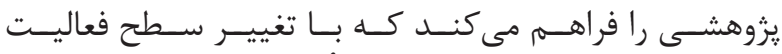

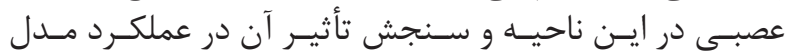

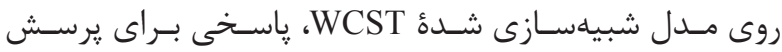

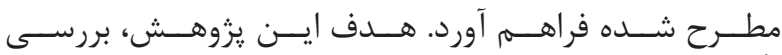

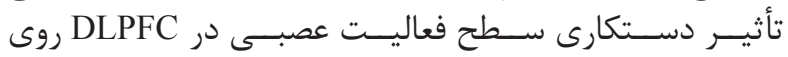

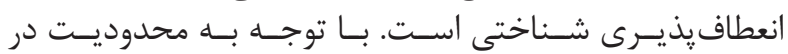

\footnotetext{
${ }^{25}$ Parallel distributed processing

${ }^{26}$ Grant and Berg

${ }^{27}$ Heaton, Chelune, Talley, Kay and Curtiss
} 


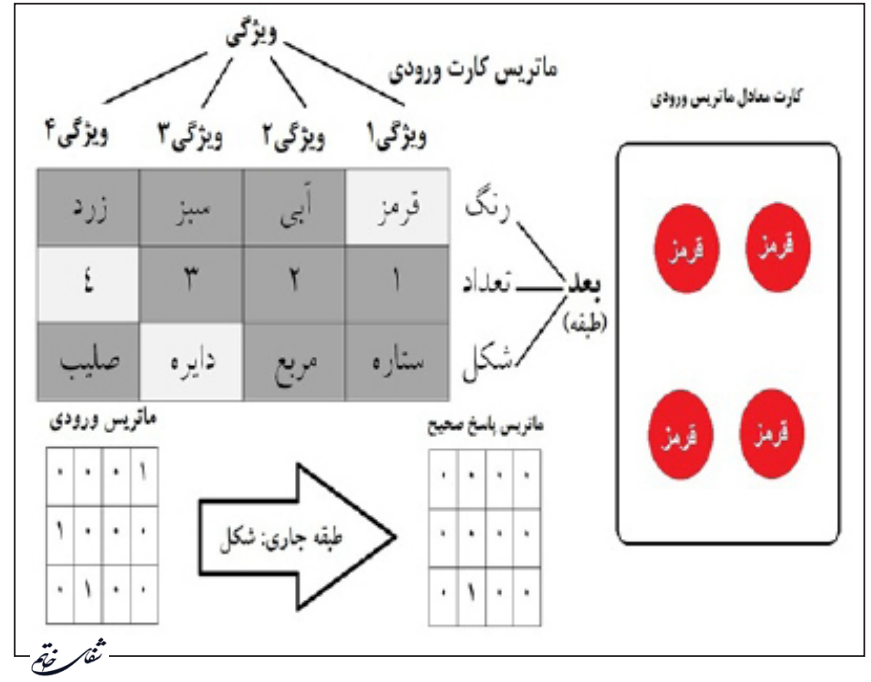

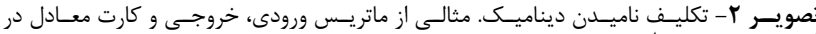

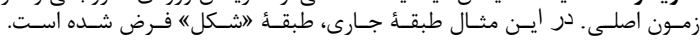

نيـز نمى توانسـد بــه صـورت همززمــان فعـال شــود.

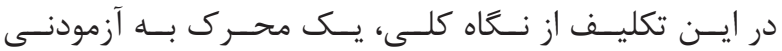

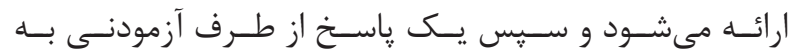

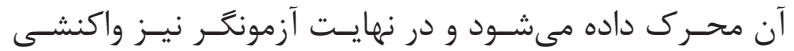

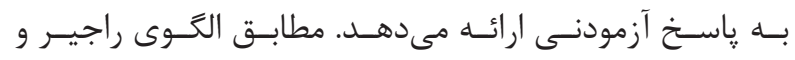

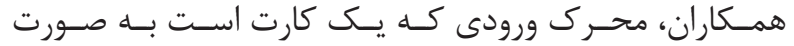

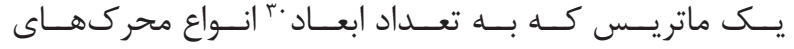

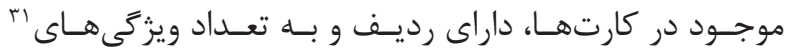

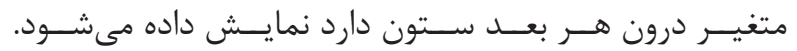

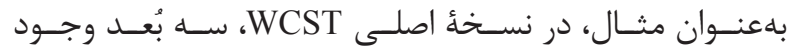

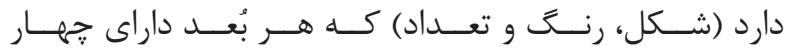

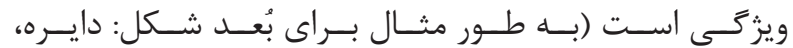
مثلـث، ســاره و و صليـبـ).

در جنيــن حالتـى بــا توجـهـه بــه اينكــه تغييــر تعـداد ابعـاد

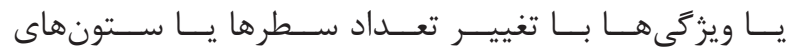

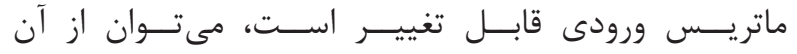

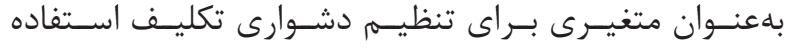

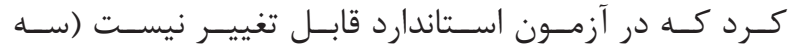

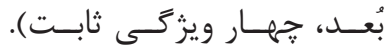
در آزمــون اسـتاندارد، كارت ورودى از بــالاى رديـف كارتهايـى

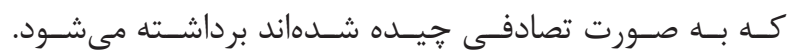

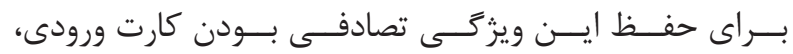

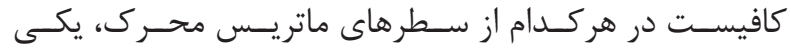

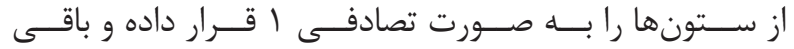

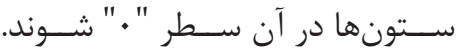

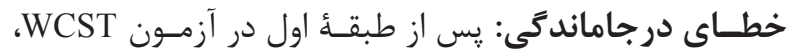

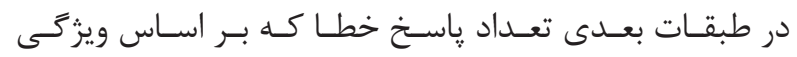

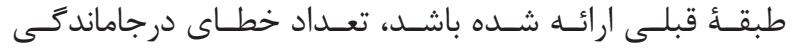

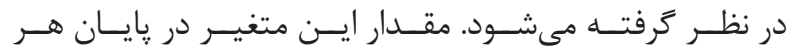

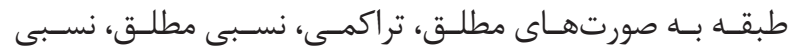

${ }^{28}$ Rougier

${ }^{29}$ Dynamic naming task

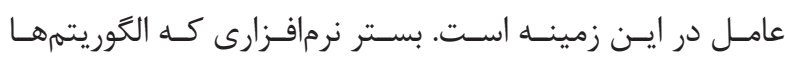

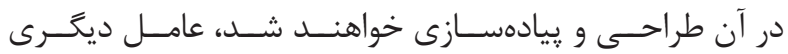

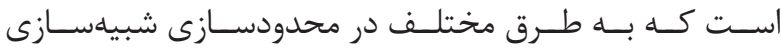

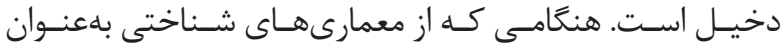

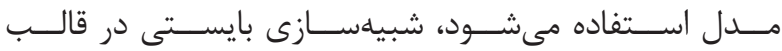

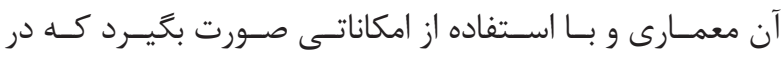

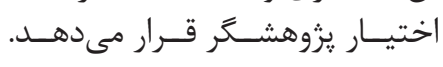

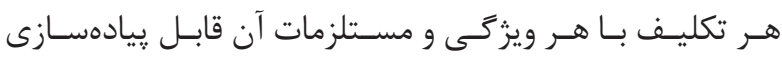

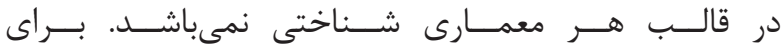

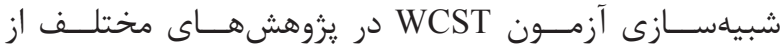

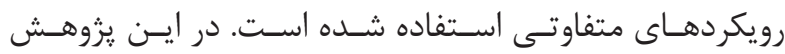

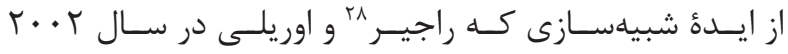

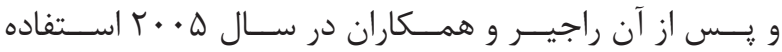

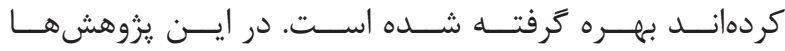

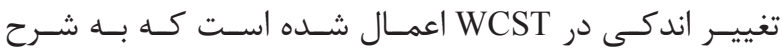

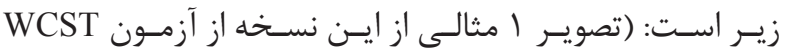

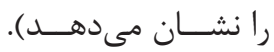

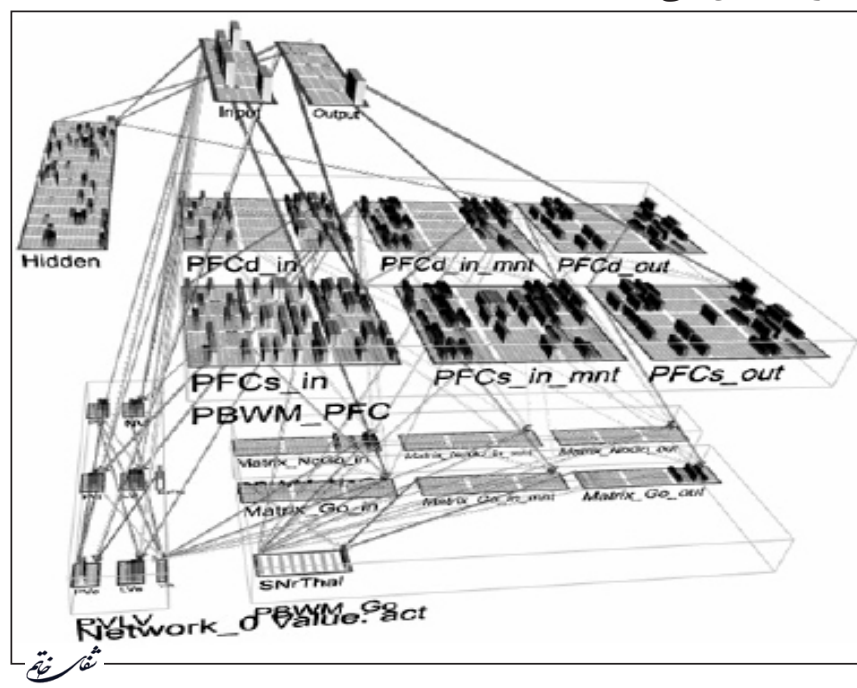

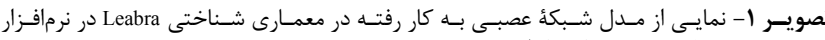
Emergent

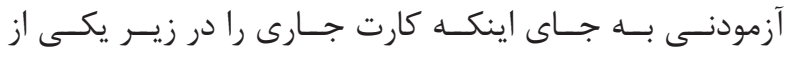

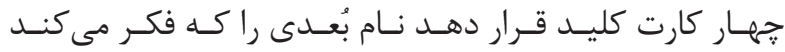

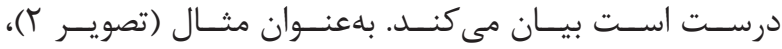

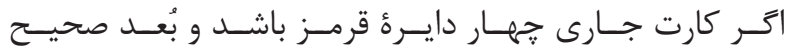

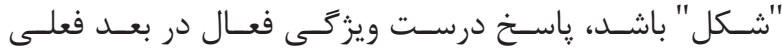

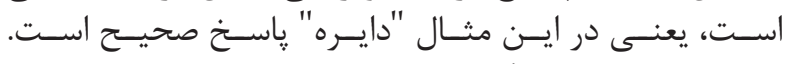

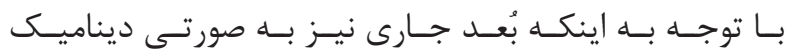

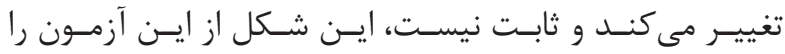

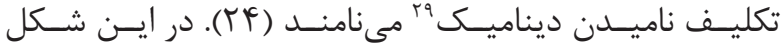

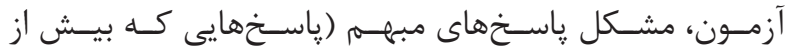

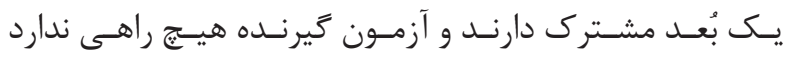

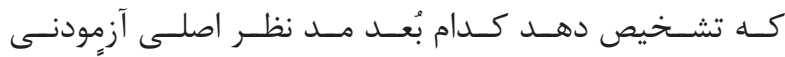

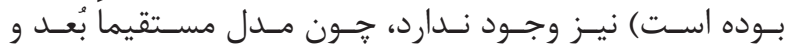

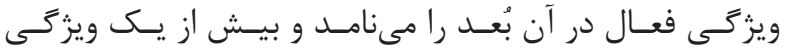

${ }^{30}$ Dimensions

${ }^{31}$ Features 


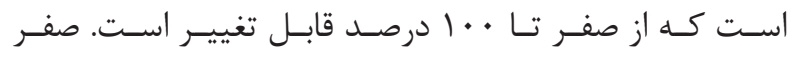

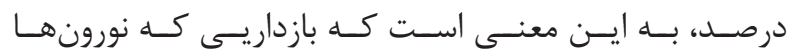

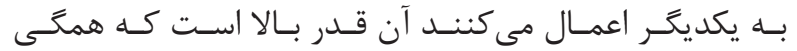

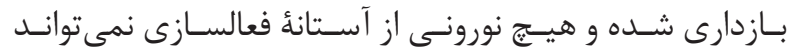

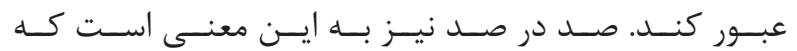

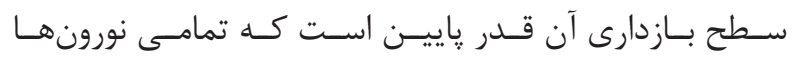

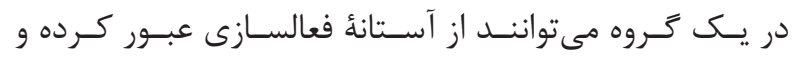

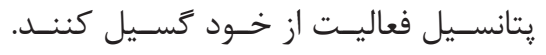

يافته ها

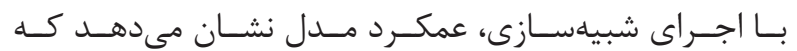

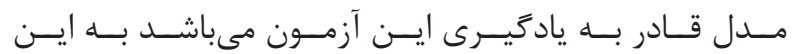

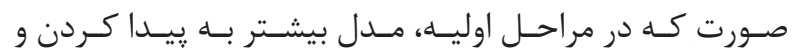

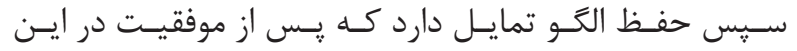

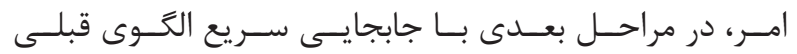

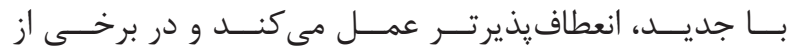

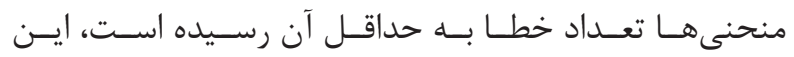

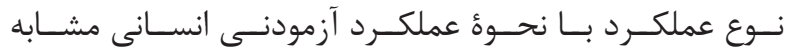

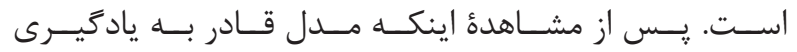

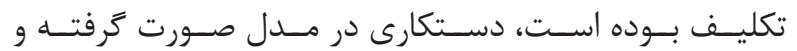

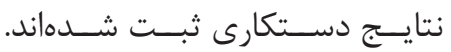

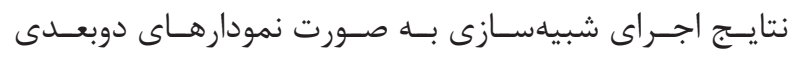

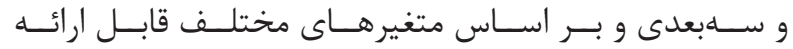

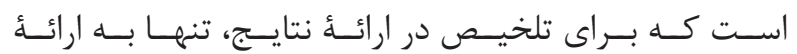

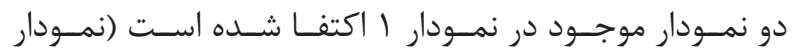

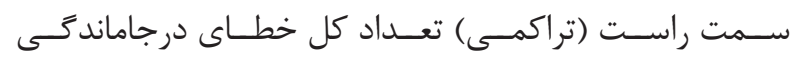

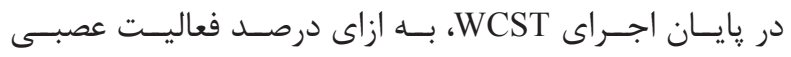

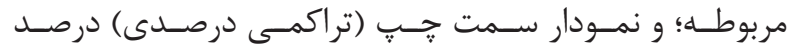

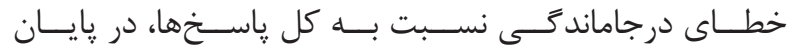

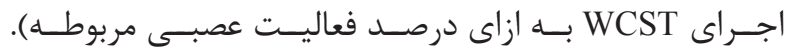

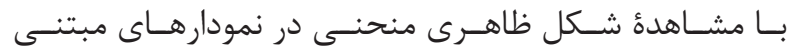

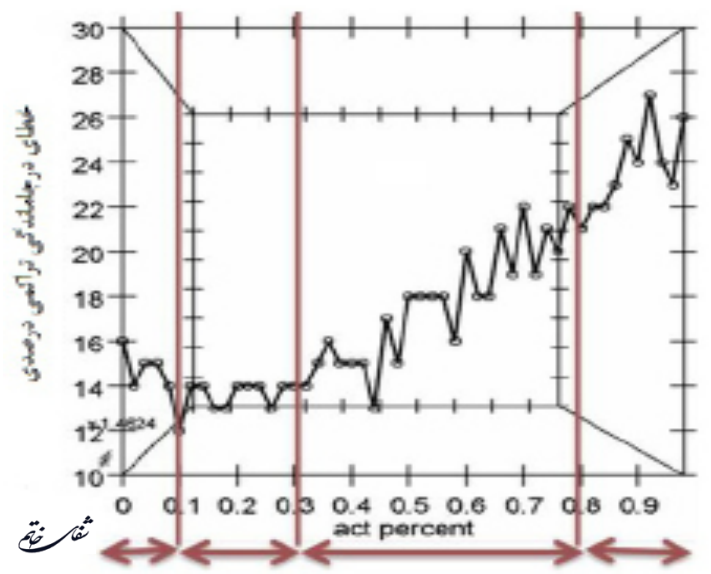

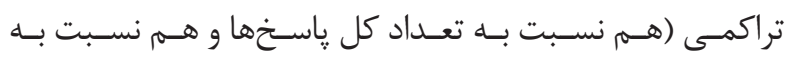

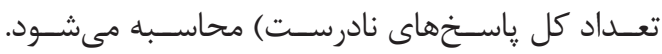

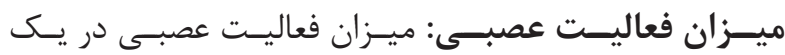

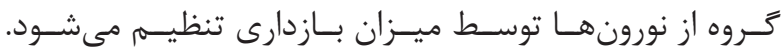

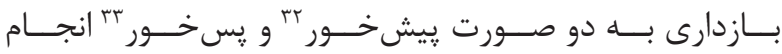

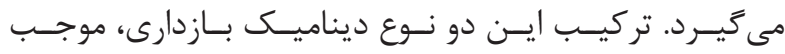

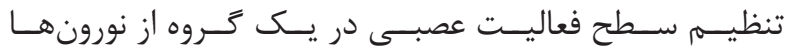

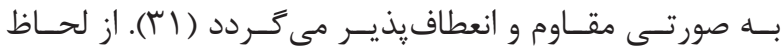

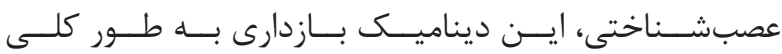

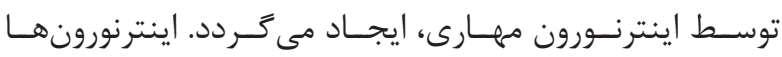

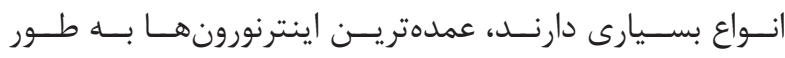

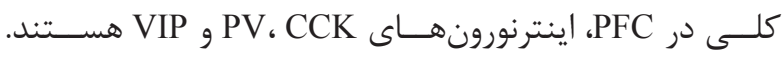

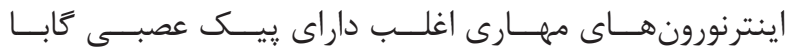

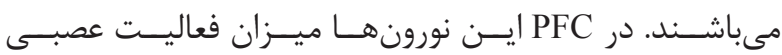

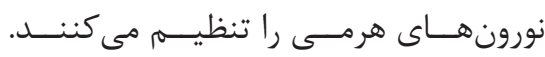

درصــد فعاليــت عصبــى: در معمـارى شــاختى Leabra،

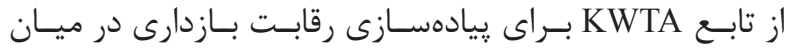

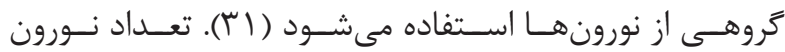

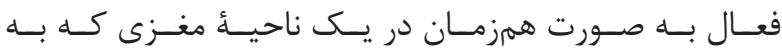

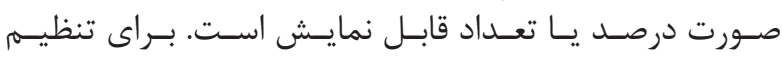

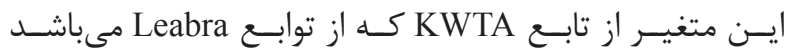

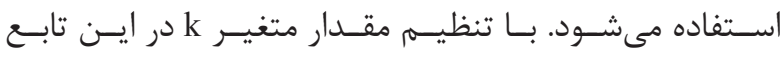

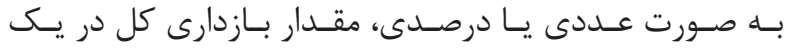

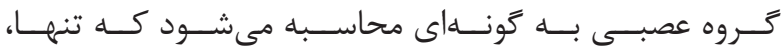

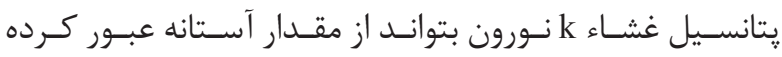

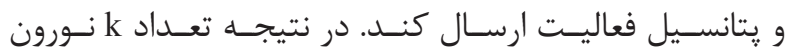

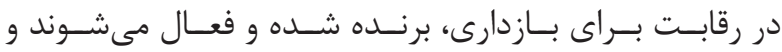

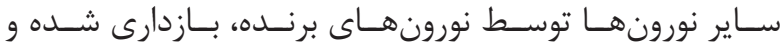

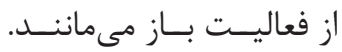
در ايـن يزوهــش از مقـدار درصـدى متغيـر k اسـتفاده شـده

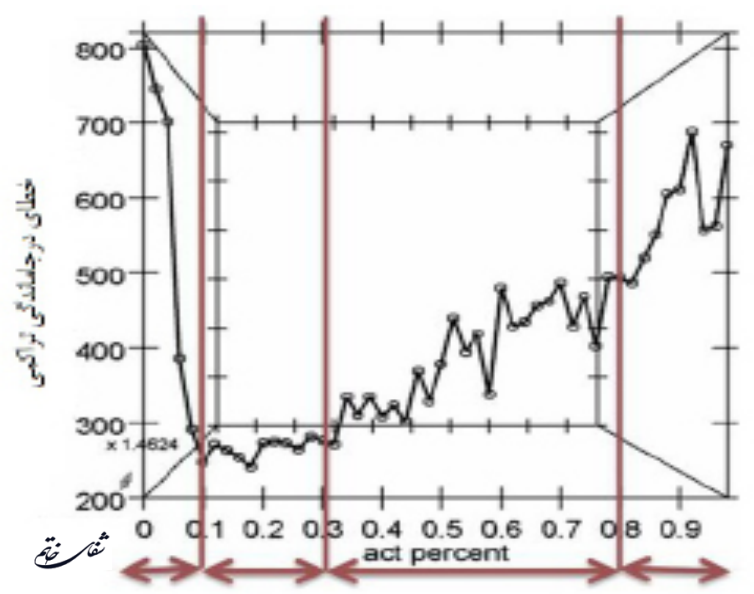

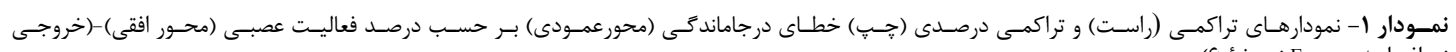

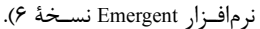

\footnotetext{
${ }^{32}$ Feedforward

${ }^{33}$ Feedback
} 


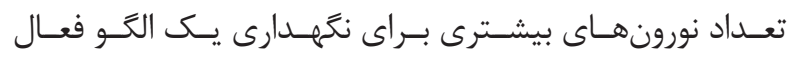

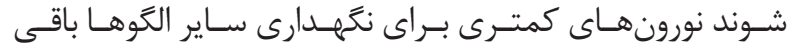

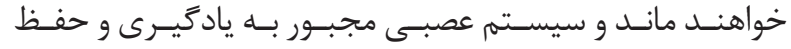

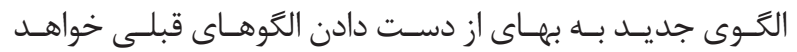

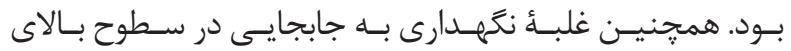

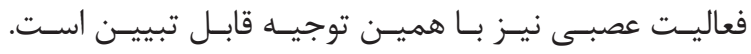

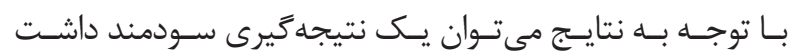

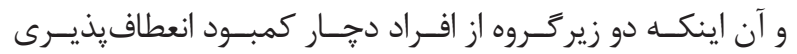

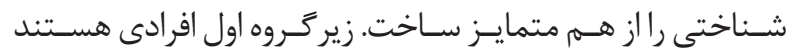

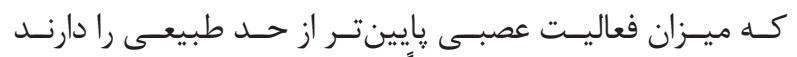

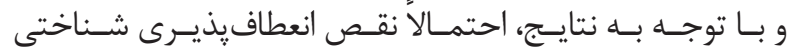

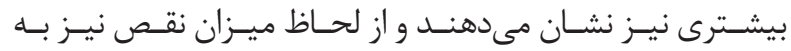

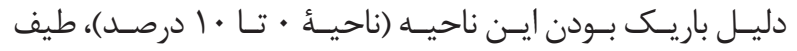

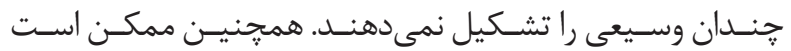

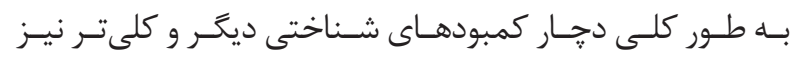

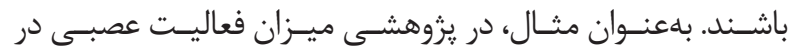

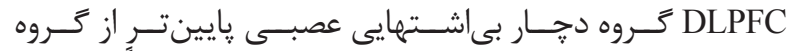

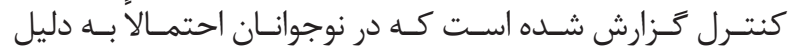

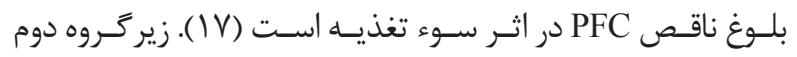

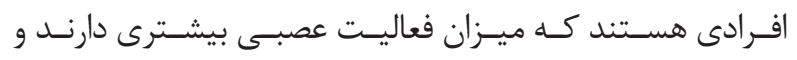

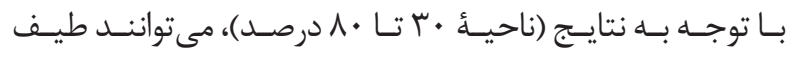

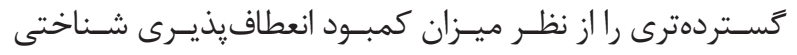

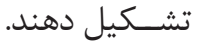

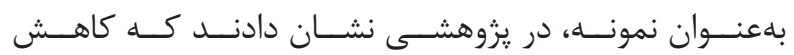

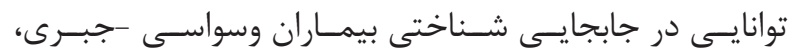

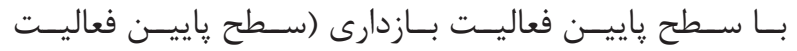

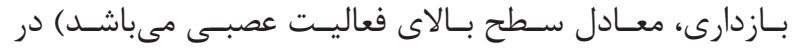

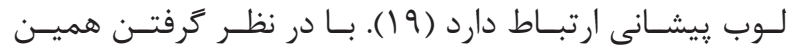

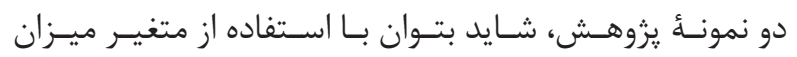

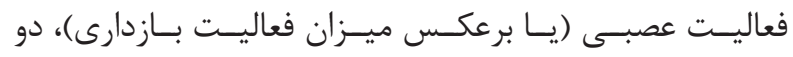

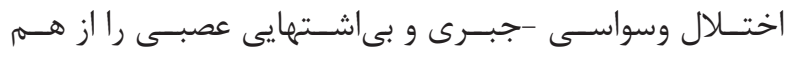

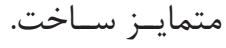

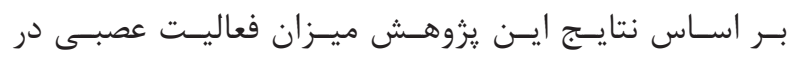

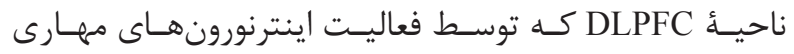

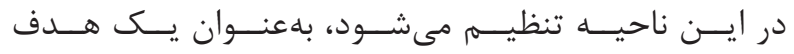

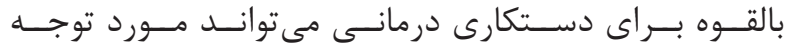

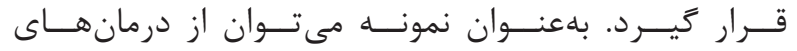

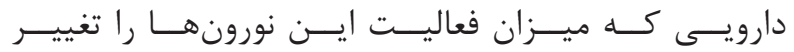

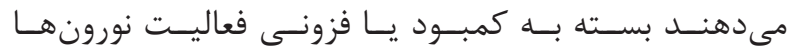

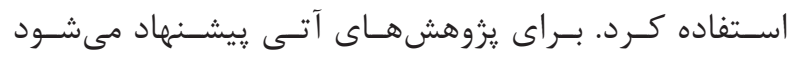

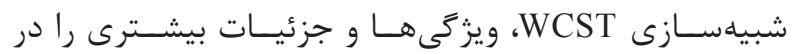

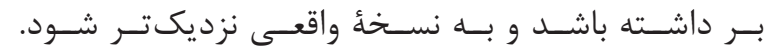

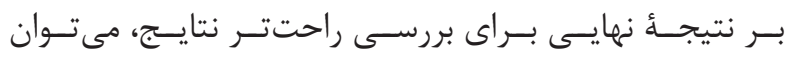

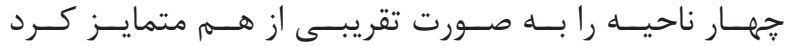

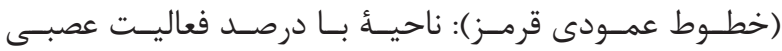

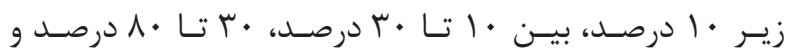
ل

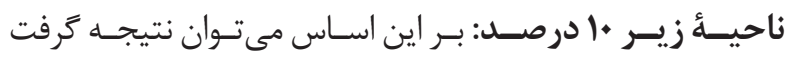

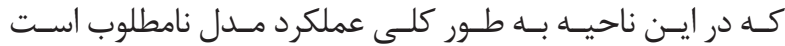

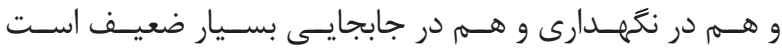

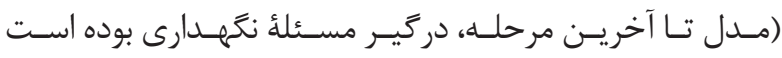

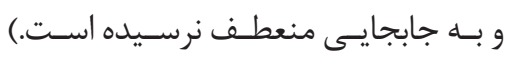

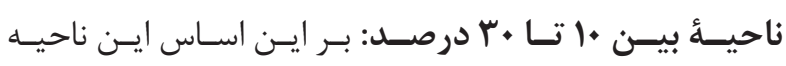

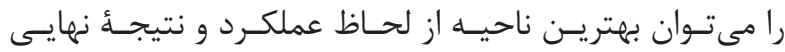
دانست.

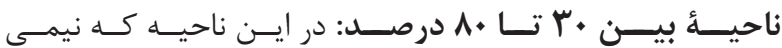

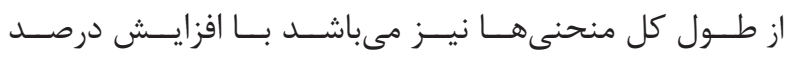

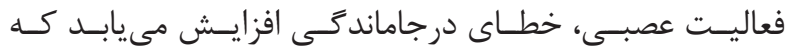

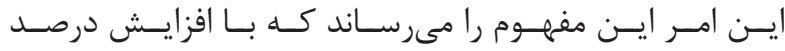

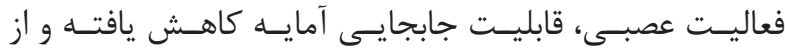

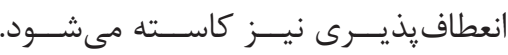

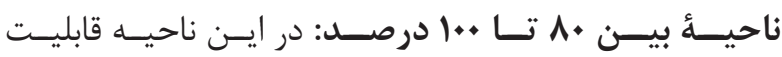

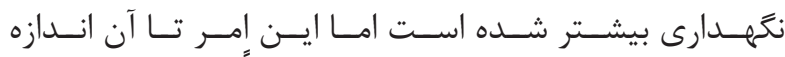

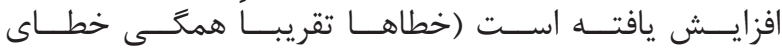

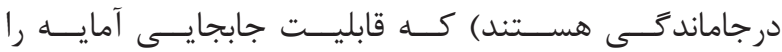

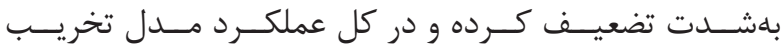

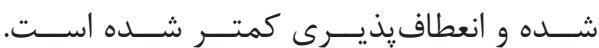

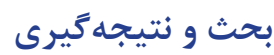

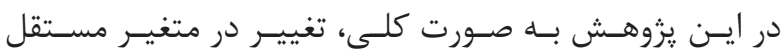

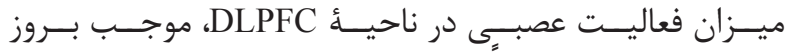

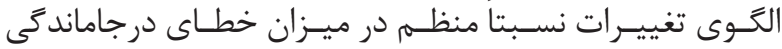

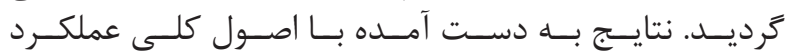

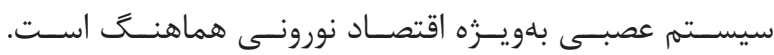

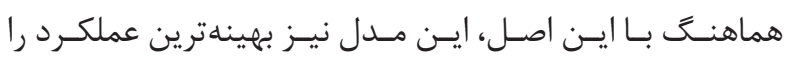

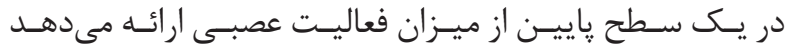

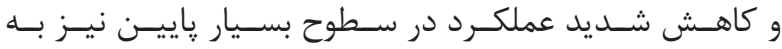

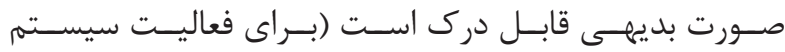

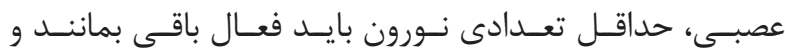

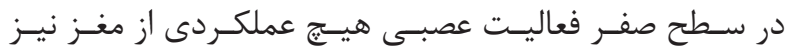

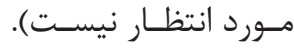

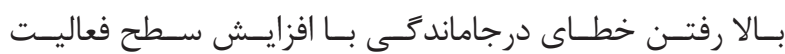

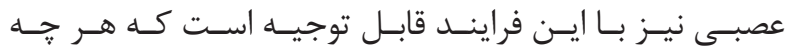


1. Scott WA. Cognitive complexity and cognitive flexibility. American Sociological Association (ASA). 1962; 25(4): 405-14.

2. Greve KW, Stickle TR, Love JM, Bianchini KJ, Stanford MS. Latent structure of the wisconsin card sorting test: a confirmatory factor analytic study. Arch Clin Neuropsychol. 2005; 20(3): 355-64.

3. Leber AB, Turk-Browne NB, Chun MM. Neural predictors of moment-to-moment fluctuations in cognitive flexibility. Proc Natl Acad Sci U S A. 2008; 105(36): 13592-7.

4. Loose R, Kaufmann C, Tucha O, Auer DP, Lange KW. Neural networks of response shifting: influence of task speed and stimulus material. Brain Res. 2006; 1090(1): 146-55.

5. Mentzel HJ, Gaser C, Volz HP, Rzanny R, Hager F, Sauer H, et al. Cognitive stimulation with the wisconsin card sorting test: functional MR imaging at $1.5 \mathrm{~T}$. Radiology. 1998; 207(2): 399-404.

6. Ko JH, Monchi O, Ptito A, Bloomfield P, Houle S, Strafella AP. Theta burst stimulation-induced inhibition of dorsolateral prefrontal cortex reveals hemispheric asymmetry in striatal dopamine release during a setshifting task-a TMS-[11C]raclopride PET study. Eur J Neurosci. 2008(10); 28: 2147-55.

7. O'Reilly RC, Hazy TE, Herd SA. The leabra cognitive architecture: how to play 20 principles with nature and win. Oxford: Oxford University Press. 2012.

8. Eylen LV, Boets B, Steyaert J, Evers K, Wagemans J, Noens I. Cognitive flexibility in autism spectrum disorder: explaining the inconsistencies? Res Autism Spectr Disord. 2011; 5(4): 1390-401.

9. Steinglass JE, Walsh BT, Stern Y. Set shifting deficit in anorexia nervosa. J Int Neuropsychol Soc. 2006; 12(3): 431-5.

10. Etchepareborda MC, Mulas F. Cognitive flexibility, an additional symptom of attention deficit hyperactivity disorder. Is it a therapeutically predictive element. Rev Neurol. 2004; 38: 97-102.

11. Demeter G, Racsmany M, Csigo K, Harsányi A, Nemeth A, Dome L. Intact short-term memory and impaired executive functions in obsessive compulsive disorder. Ideggyogy Sz. 2013; 66(1-2): 35-41.
12. Tchanturia K, Davies H, Roberts M, Harrison A, Nakazato M, Schmidt U, et al. Poor cognitive flexibility in eating disorders: examining the evidence using the wisconsin card sorting task. plos one. 2012;7(1): e28331. doi: 10.1371/journal.pone.0028331.

13. Chamberlain SR, Blackwell AD, Fineberg NA, Robbins TW, Sahakian BJ. The neuropsychology of obsessive compulsive disorder: the importance of failures in cognitive and behavioural inhibition as candidate endophenotypic markers. Neurosci Biobehav Rev. 2005; 29(3): 399-419.

14. Kriete T, Noelle DC. Impaired cognitive flexibility and intact cognitive control in autism: a computational cognitive neuroscience approach. Top Cogn Sci. 2005; 1: 1190-5.

15. Buhren K, Mainz V, Herpertz-Dahlmann B, Schafer K, Kahraman-Lanzerath B, Lente C, et al. Cognitive flexibility in juvenile anorexia nervosa patients before and after weight recovery. J Neural Transm (Vienna). 2012; 119(9): 1047-57.

16. StalnakerTA, TakahashiY, Roesch MR, Schoenbaum G. Neural substrates of cognitive inflexibility after chronic cocaine exposure. Neuropharmacology. 2009; 56(1): 63-72.

17. Sato Y, Saito N, Utsumi A, et al. Neural basis of impaired cognitive flexibility in patients with anorexia nervosa. plos one. 2013; 8(5): e61108. doi: 10.1371/ journal.pone.0061108.

18. Dupuy M, Rouillon F, Bungener C. The role of inhibition in obsessional-compulsive disorders. Encep. 2013; 39(1): 44-50.

19. Sanz M, Molina V, Martin-Loeches M, Calcedo A, Rubia FJ. Auditory p300 event related potential and serotonin reuptake inhibitor treatment in obsessivecompulsive disorder patients. Psychiatry Res. 2001; 101(1): 75-81.

20. Berman KF, Ostrem JL, Randolph C, Gold J, Goldberg TE, Coppola R, et al. Physiological activation of a cortical network during performance of the wisconsin card sorting test: a positron emission tomography study. Neuropsychologia. 1995; 33(8): 1027-46.

21. Cabeza R, Nyberg L. Imaging cognition II: an empirical review of 275 PET and fMRI studies. J Cogn Neurosci. 2000; 12(1): 1-47. 


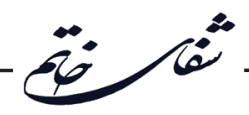

22. Monchi O, Petrides M, Petre V, Worsley K, Dagher A. Wisconsin card sorting revisited: distinct neural circuits participating in different stages of the task identified by event-related functional magnetic resonance imaging. J Neurosci. 2001; 21(19): 7733-41.

23. Frank M, Loughry B, O’Reilly R. Interactions between frontal cortex and basal ganglia in working memory: a computational model. Cogn Affect Behav Neurosci. 2001; 1(2): 137-60.

24. O'Reilly RC, Frank MJ. Making working memory work: a computational model of learning in the prefrontal cortex and basal ganglia. Neural Comput. 2006; 18(2): 283-328.

25. Ron S. The cambridge handbook of computational psychology. New York: Cambridge University Press; 2008.

26. Rumelhart DE, McClelland JL, Group tPR. Parallel distributed processing: explorations in the microstructure of cognition. Cambridge: MIT Press; 1986.

27. Marcus GF. The algebraic mind: integrating connectionism and cognitive science (learning, development, and conceptual change). Cambridge: MIT Press; 2003.

28. McClelland JL. The place of modeling in cognitive science. Top Cogn Sci. 2009; 1: 11-38.

29. Ashby FG, Helie S. A tutorial on computational cognitive neuroscience: modelingthe neurodynamics of cognition. J Math Psychol. 2011; 55(4)1: 273-89.

30. O’Reilly RC. Six principles for computational cognitive neuroscience models. Trends Cogn Sci. 1998; 11: $1241-8$.

31. O’Reilly RC, Munakata Y. Computational explorations in cognitive neuroscience:understanding the mind by simulating the brain. Cambridge: MIT Press; 2000.

32. Hazy TE, Frank MJ, O’Reilly RC. Neural mechanisms supporting acquired phasic dopamine responses in learning: an integrative synthesis. Neurosci Biobehav Rev. 2010; 34(5): 701-20.

33. Frank MJ, Munakata Y, Hazy T, O’Reilly R. Computational Cognitive Neuroscience. Kindle Store: wikibook; 2012.

34. Stemme A, Deco G, Busch A. The neuronal dynamics underlying cognitive flexibility in set shifting tasks. J Comput Neurosci. 2007; 23: 313-31.

35. Kaplan GB, Genc I, Guzelis C. Simple neural network system for wisconsin card sorting test. Conf Proc IEEE Eng Med Biol Soc. 2001; 1: 1094-687.

36. Kaplan G, Sengor N, Gurvit H, Genc I, Guzelis C. A composite neural network model for perseveration and distractibility in the Wisconsin card sorting test. Neural Netw. 2006; 19(4): 375-87.

37. Rougier NP, O’Reilly RC. Learning representations in a gated prefrontal cortex model of dynamic task switching. Cognitive Science. 2002; 26(4): 504-20.

38. O'Reilly RC, Noelle DC, Braver TS, Cohen JD. Prefrontal cortex and dynamic categorization tasks: representational organization and neuromodulatory control. Cereb Cortex. 2002; 12(3): 246-57.

39. Rougier NP, Noelle DC, Braver TS, Cohen JD, O'Reilly RC. Prefrontal cortex and flexible cognitive control: rules without symbols. PNAS. 2005; 102(20): 7338-43.

40. Nyhust E, Barcelo F. The wisconsin card sorting test and the cognitive assessment of prefrontal executive functions: a critical update. Brain Cogn. 2009; 71(3): 437-51.

41. Aisa b, Mingus B, O'Reilly r. The emergent neural modeling system. Neural Netw. 2008; 21(8): 1146-52.

42. Berg EA. A simple objective technique for measuring flexibility in thinking. J Gen Psychol. 1948; 39: $15-22$. 